\title{
Glutathione-S-Transferase to Creatinine Ratio Measurement
}

National Cancer Institute

\section{Source}

National Cancer Institute. Glutathione-S-Transferase to Creatinine Ratio Measurement. NCI Thesaurus. Code C79435.

The determination of the ratio of glutathione-s-transferase compared to creatinine present in a sample. The measurement may be expressed as a ratio or percentage. 